\title{
Lattice study of the infrared behavior of QCD Green's functions in Landau gauge
}

\author{
A. Sternbeck ${ }^{* \dagger}$ \\ Centre for the Subatomic Structure of Matter (CSSM), School of Chemistry \& Physics, \\ University of Adelaide, SA 5005, Australia \\ E-mail: andre.sternbeck@adelaide.edu.de

\section{E.-M. Ilgenfritz and M. Müller-Preussker} \\ Humboldt-Universität zu Berlin, Institut für Physik, 12489 Berlin, Germany \\ E-mail: ilgenfri@physik.hu-berlin.de, mmp@physik.hu-berlin.de
}

\section{A. Schiller}

Universität Leipzig, Institut für Theoretische Physik, 04109 Leipzig, Germany

E-mail: Arwed.Schiller@itp.uni-leipzig.de

\section{L. Bogolubsky}

Joint Institute for Nuclear Research, 141980 Dubna, Russia

E-mail: bogolubsajinr.ru

\begin{abstract}
We summarize the current status of our numerical results for the gluon and ghost propagators and for the Kugo-Ojima confinement parameter in quenched $S U(3)$ lattice Landau gauge theory. The data for the propagators are compared to our results obtained in the case of full QCD, simulated using two flavors of dynamical clover-improved Wilson fermions. We demonstrate that the infrared behavior of the ghost propagator is consistent with the Kugo-Ojima confinement criterion. Explicit violation of reflection positivity by the gluon propagator is shown. Additionally, we present results of a running coupling constant both at low and at large momenta.
\end{abstract}

XXIV International Symposium on Lattice Field Theory

July 23-28 2006

Tucson, Arizona, US

\footnotetext{
* Speaker.

${ }^{\dagger}$ Address until August 31, 2006: Humboldt-Universität zu Berlin, Institut für Physik, D-12489 Berlin, Germany.
} 


\section{Introduction}

In the last years progress has been made to improve our understanding of QCD Green's functions in different gauges. This is important, because it might allow us someday to describe all hadronic features in the continuum directly in terms of those functions. A deeper knowledge of QCD Green's functions would help us also to understand the basics of nonperturbative phenomena like gluon and quark confinement or dynamical chiral symmetry breaking from first principles. For example, the realization of the Kugo-Ojima confinement scenario [1] in QCD in covariant gauges is encoded in the infrared behavior of the gluon and ghost 2-point functions. Therefore, the investigation of QCD Green's functions is not only of interest for a coherent description of hadronic states but also for an understanding of confinement.

At large (Euclidean) momenta QCD Green's functions can be described in terms of perturbation theory. However, at intermediate and low momenta nonperturbative methods are indispensable to arrive at a complete picture. There are different approaches to deal with nonperturbative QCD, but they all have their own limitations. Therefore, a comparison of results is important. For example, studies of truncated Dyson-Schwinger equations (DSEs) for the gluon and ghost propagators in Landau gauge came to the conclusion that at very low momenta these propagators are governed by power laws with interrelated exponents which then results in a coupling constant running to a nontrivial infrared fixed point [3]. This infrared behavior has been confirmed independently by studies of exact renormalization group equations [4] and investigations based on the Fokker-Planck-type diffusion equation of stochastic quantization [5]. At intermediate momenta, however, predictions e.g. based on DSE studies are uncertain due to the truncations involved, but in this region lattice Monte Carlo (MC) simulations can help.

\section{Lattice results for the gluon and ghost propagators}

We have performed MC simulations in the quenched approximation of $S U(3)$ lattice gauge theory at four values of the coupling constant $(\beta=5.7,5.8,6.0,6.2)$ using the standard gluonic Wilson action. Thereby, the lattice size ranged between $16^{4}$ and $56^{4}$. To study also the influence of fermions we have analyzed gauge field configurations generated with $N_{f}=2$ dynamical flavors of clover-improved Wilson fermions using the same gauge action ${ }^{1}$. All gauge configurations have been fixed to Landau gauge by maximizing the Landau gauge functional

$$
F_{U}[g]=\frac{1}{4 V} \sum_{x} \sum_{\mu=1}^{4} \mathfrak{R e} \operatorname{Tr}^{g} U_{x, \mu} \quad \text { with } \quad{ }^{g} U_{x, \mu}=g_{x} U_{x, \mu} g_{x+\hat{\mu}}^{\dagger}
$$

using either over-relaxation or Fourier-accelerated gauge-fixing. Of course, the maxima are not unique, but we will neglect the influence of Gribov copies in the following. The reader is referred to Ref. [6] for a more systematic account of the Gribov ambiguity in this context.

After gauge-fixing we have calculated the gluon and ghost propagators on all (gauge-fixed) configurations. The definition of both Green's functions and their dressing functions is standard and our notation can be found in detail in Ref. [7]. To reduce finite volume and discretization effects we have applied cone and cylinder cuts to our data [8].

\footnotetext{
${ }^{1}$ Those configurations were provided to us by the QCDSF collaboration.
} 
Considering first the quenched case, in Fig. 1 we show data for the ghost dressing function $J\left(q^{2}\right)$ (renormalized at $\mu=4 \mathrm{GeV}$ ) as a function of the momentum $q^{2}$. As expected the ghost dressing function seems to diverge at vanishing momentum. We have tried to fit the infrared power law as expected from DSE studies to our data. However, within the region of lower momenta a power law does not describe the data that well. The ghost dressing function seems to increase logarithmically as the momenta become small. In any case, the infrared exponent extracted from the fit, $\kappa \approx 0.2$, is much smaller then it is expected to be, namely $\kappa \approx 0.59[5,9]$. With respect to our data for the gluon propagator, shown in Fig. 2, we think that we are still not in a region of momenta where the mentioned infrared power law can be verified. For this the gluon propagator $D$ has to vanish at zero momentum which cannot be concluded from our current data (see Fig. 2). To what extent finite volume effects can be blamed for such a (different) behavior found on the lattice needs to be clarified in future yet.

To improve our understanding of lattice Landau gauge theory we have also studied the influence of (clover-improved) Wilson fermions on the ghost and gluon propagators. We find that the ghost propagator stays almost unchanged if fermions are added to the gauge action. In contrast to this, fermions affect the gluon propagator at large and intermediate momenta, in particular where the gluon propagator exposes its characteristic enhancement compared to the free propagator. To illustrate this, in Fig. 3 we show our data for the gluon dressing function obtained for quenched and full QCD.

As a part of the project we also performed simulations at $\beta=6.0$ using large asymmetric lattice sizes, namely $16^{3} \times 128$ and $24^{3} \times 128$. By comparing the data obtained on these lattices for

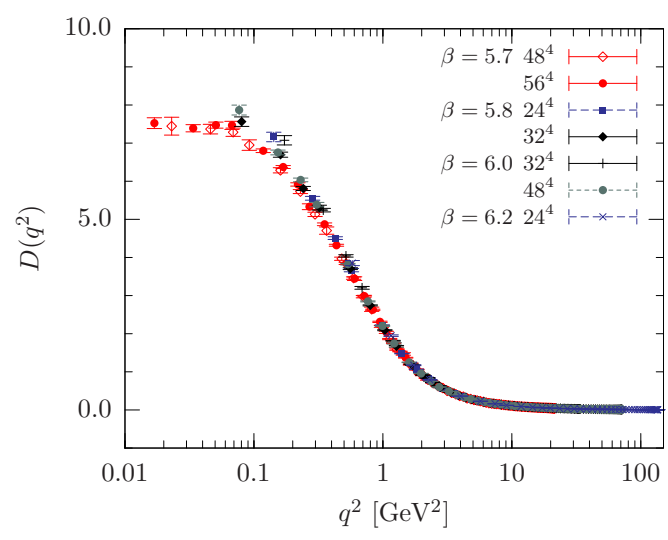

Figure 2: The (quenched) gluon propagator $D$ as a function of $q^{2}$.

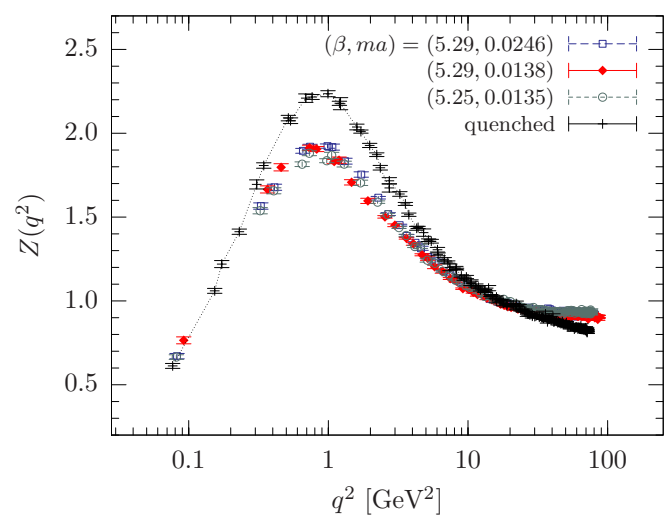

Figure 3: The gluon dressing function $Z$ for the quenched and unquenched case as a function of $q^{2}$ 


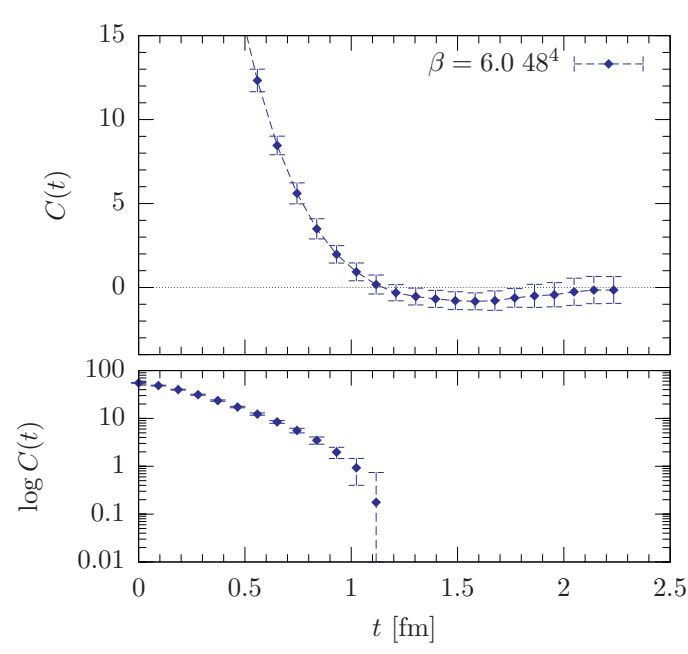

Figure 4: The upper panel shows the zero threemomentum propagator $C(t)$ of the gluon fields in quenched QCD at $\beta=6.0$ for a $48^{4}$ lattice as a function of time. In the lower panel the same data are shown, however, as $\log C(t)$ for $C(t)>0$.

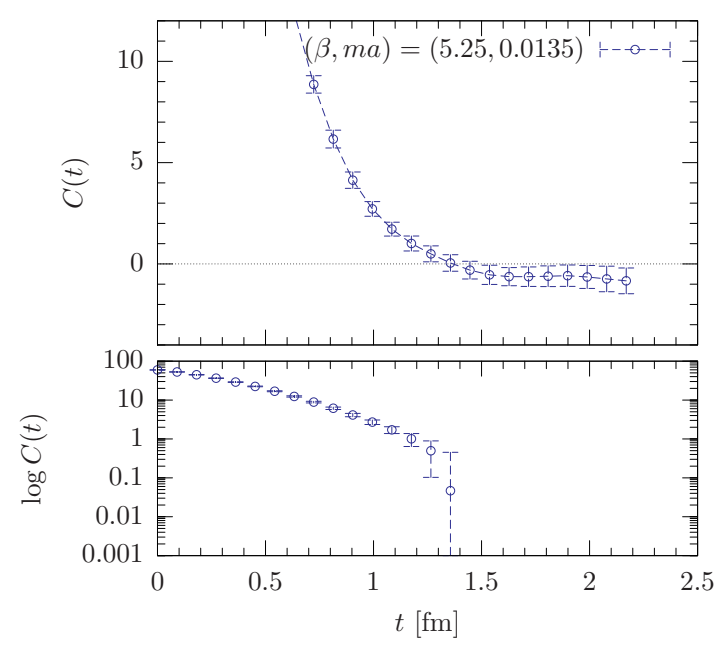

Figure 5: The same as in the left figure, however, for the gluon propagator in full QCD on a $24^{3} \times 48$ lattice for one particular mass ma.

the ghost and gluon propagators to our results using symmetric lattices $\left(\right.$ e.g. $\left.48^{4}\right)$ we found large systematic effects at low momentum due to the asymmetry involved, in particular, for the lowest on-axis momenta along the elongated 'time' direction. ${ }^{2}$ It is not excluded that sensible results at lower momenta for the gluon and ghost propagators can be extracted from asymmetric lattices by careful extrapolations. Attempts in that directions are made e.g. in [10].

\section{Confinement criterion I: Violation of reflection positivity}

In view of the general objectives of this project it is also important to check whether our data for the propagators satisfy necessary criteria for confinement. For example, the gluon propagator has to violate reflection positivity, because otherwise gluons could be interpreted in terms of stable particle states. ${ }^{3}$ To check whether reflection positivity is violated by the gluon propagator we have calculated the space-time (lattice) correlator at zero spatial momentum as function of time

$$
C(t):=\frac{1}{\sqrt{L_{T}}} \sum_{k_{4}=0}^{L_{T}-1} D\left(\overrightarrow{0}, k_{4}\right) \exp \left\{\frac{2 \pi i k_{4} t}{L_{T}}\right\}
$$

using our data for the gluon propagator $D\left(\overrightarrow{0}, k_{4}\right)$. The result for the quenched case $\left(\beta=6.0,48^{4}\right.$ lattice) is shown in Fig. 4. Obviously, reflection positivity is violated in a finite range of $t$. The same holds in the unquenched case as can be seen in Fig. 5, even though the lattice size in this case is too small such that the data do not bend over towards zero at larger $t$ as in the quenched case (Fig. 4). Note that we have seen this happening also in our quenched data at $\beta=6.0$ using a $32^{4}$ lattice (not shown).

\footnotetext{
${ }^{2}$ For more details about this but also other systematic effects have a look at the Ph.D. thesis [7] of one of us.

${ }^{3}$ Note that the ghost propagator violates reflection positivity trivially and hence ghosts are explicitly unphysical.
} 


\section{Confinement criteria II: The Kugo-Ojima confinement parameter}

Another criterion for confinement for QCD in covariant gauges was given by Kugo and Ojima long time ago [1]. According to their scenario colored asymptotic states, if any, cannot be detected in the physical subspace due to the quartet mechanism. This is realized if a function $u\left(p^{2}\right)$, defined as $[2,11]$

$$
\int d^{4} x e^{i p(x-y)}\left\langle D_{\mu}^{a e} c^{e}(x) g f^{b c d} A_{v}^{d}(y) \bar{c}^{c}(y)\right\rangle=:\left(\delta^{\mu v}-\frac{p_{\mu} p_{v}}{p^{2}}\right) \delta^{a b} u\left(p^{2}\right),
$$

has the zero-momentum limit

$$
\mathrm{u}:=\lim _{p^{2} \rightarrow 0} u\left(p^{2}\right)=-1 .
$$

In Landau gauge this limit is connected to an infrared diverging ghost dressing function $J$ through [2]

$$
J\left(p^{2}\right)=\frac{1}{1+u\left(p^{2}\right)+p^{2} v\left(p^{2}\right)} \stackrel{p^{2} \rightarrow 0}{\longrightarrow} \frac{1}{1+\mathrm{u}} .
$$

Here $v\left(p^{2}\right)$ is an unknown function of $p^{2}$ (see [2] for a definition). We have made an attempt to confirm the realization of the limit in Eq. (4.2), not only by giving numerical evidence for a diverging ghost dressing function (see above), but also by estimating $u\left(p^{2}\right)$ itself at different momenta $p^{2}$ in

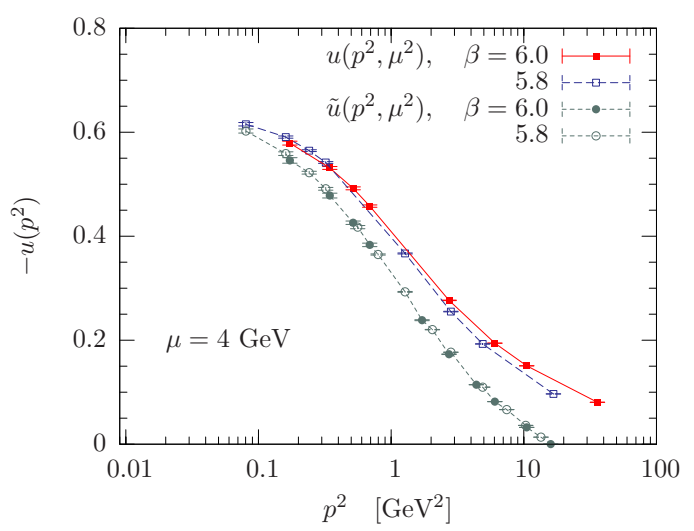

Figure 6: Data of $u\left(p^{2}, \mu^{2}\right)$ at $\beta=5.8$ and 6.0 are shown using squares. Additionally, data of $\tilde{u}$ are shown at the same $\beta$ values (circles). All data refer to the same quenched configurations on a $32^{4}$ lattice and are renormalized at $\mu=4 \mathrm{GeV}$ as described in [7]. Lines are drawn to guide the eye. our lattice simulations ${ }^{4}$. Our estimates of $u\left(p^{2}\right)$ renormalized at $\mu=4 \mathrm{GeV}$, are shown in Fig. 6 as a function of $p^{2}$. There we also show data of the ghost dressing function (renormalized at the same $\mu$ ) in the form of an asymptote $\tilde{u}$ defined as

$$
\tilde{u}\left(p^{2}, \mu^{2}\right):=\frac{1}{J\left(p^{2}, \mu^{2}\right)}-1 .
$$

Obviously, $\tilde{u}\left(p^{2}\right)$ and $u\left(p^{2}\right)$ are different at finite momentum, but according to Eq. (4.3) $u\left(p^{2}\right)$ has to reach asymptotically $\tilde{u}\left(p^{2}\right)$ in the zero momentum limit. From our data shown in Fig. 6 we can confirm that the difference $\left|u\left(p^{2}\right)-\tilde{u}\left(p^{2}\right)\right|$ diminishes with decreasing momentum, even though an extrapolation of the given data to vanishing momentum is difficult to perform. For this, the explicit momentum dependence of $u$ has to be known. Since $u\left(p^{2}\right)$ seems to continuously approach $\tilde{u}\left(p^{2}\right)$ with decreasing momentum and, by definition, $\tilde{u}\left(p^{2}\right)$ is minus one at vanishing momentum for a diverging ghost dressing function our studied momentum range of $u$ at least does not exclude the expected zero momentum limit (see Eq. (4.2)).

\section{The running coupling constant}

Given the dressing functions of gluon and ghost propagators a running coupling constant

$$
\alpha_{s}\left(q^{2}\right)=\alpha_{s}\left(\mu^{2}\right) Z\left(q^{2}, \mu^{2}\right) J^{2}\left(q^{2}, \mu^{2}\right)
$$

\footnotetext{
${ }^{4}$ For details on our estimation and renormalization of $u\left(p^{2}\right)$ on the lattice we refer to Ref. [7].
} 
based on the ghost-gluon-vertex can be calculated. This definition relies on the assumption that in Landau gauge this vertex stays bare also beyond perturbation theory. We have given numerical evidence in quenched and unquenched $S U(3)$ lattice gauge theory confirming that the vertex renormalization constant $\widetilde{Z}_{1} \approx 1$ in a (asymmetric) MOM scheme where the gluon momentum equals zero (see [7, 12]). Similar results indicating this, directly and indirectly, were presented in lattice studies of quenched $S U$ (2) gauge theory [13], but also within the DSE approach [14]. Thus the definition of $\alpha_{s}\left(q^{2}\right)$ according to Eq. (5.1) is valid at least in

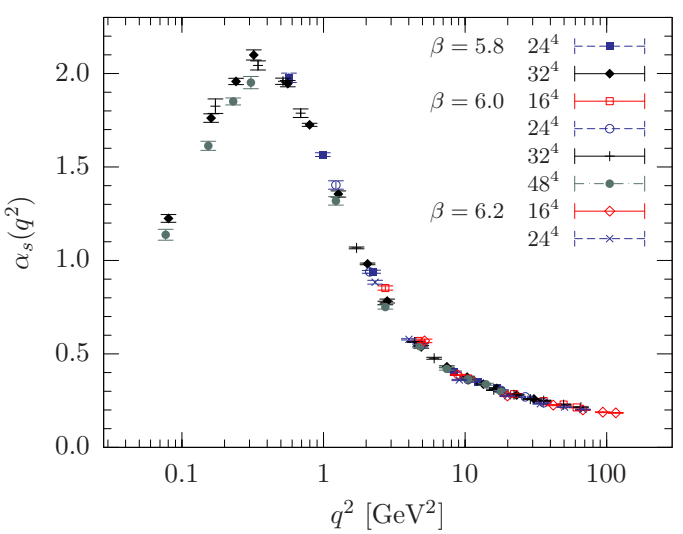

Figure 7: The running coupling $\alpha_{s}\left(q^{2}\right)$ as a function of the momentum $q^{2}$. that scheme.

In Fig. 7 we show our current data of $\alpha_{s}$ in the quenched case. One clearly sees it increasing towards lower momenta as long as $q^{2}>0.3 \mathrm{GeV}^{2}$. After passing a maximum at $q^{2} \approx 0.3 \mathrm{GeV}^{2}$ it decreases. We have found the same behavior on our sets of dynamical gauge configurations (see e.g. Fig. 4 in Ref. [12]). Therefore, on the basis of the present data we cannot confirm $\alpha_{s}\left(q^{2}\right)$ to approach a non-trivial infrared fix-point, even less monotonously from below as expected from DSE studies. The reason for this is still unclear, however, in the light of the DSE results on a torus this might be a finite volume effect which we are unable to resolve at the present stage.

Apart from the low-momentum region it is also interesting to look at $\alpha_{s}\left(q^{2}\right)$ at larger momenta and to fit the corresponding 1-loop and 2-loop expressions to the data. This is shown in Fig. 8 for the quenched (1.h.s.) and unquenched case (r.h.s.). Obviously, the data follow the one and twoloop expression in the marked interval. However, large discretization errors are visible. Therefore, simulations at smaller lattice spacings are worthwhile in order to get more reliable values of $\Lambda$.
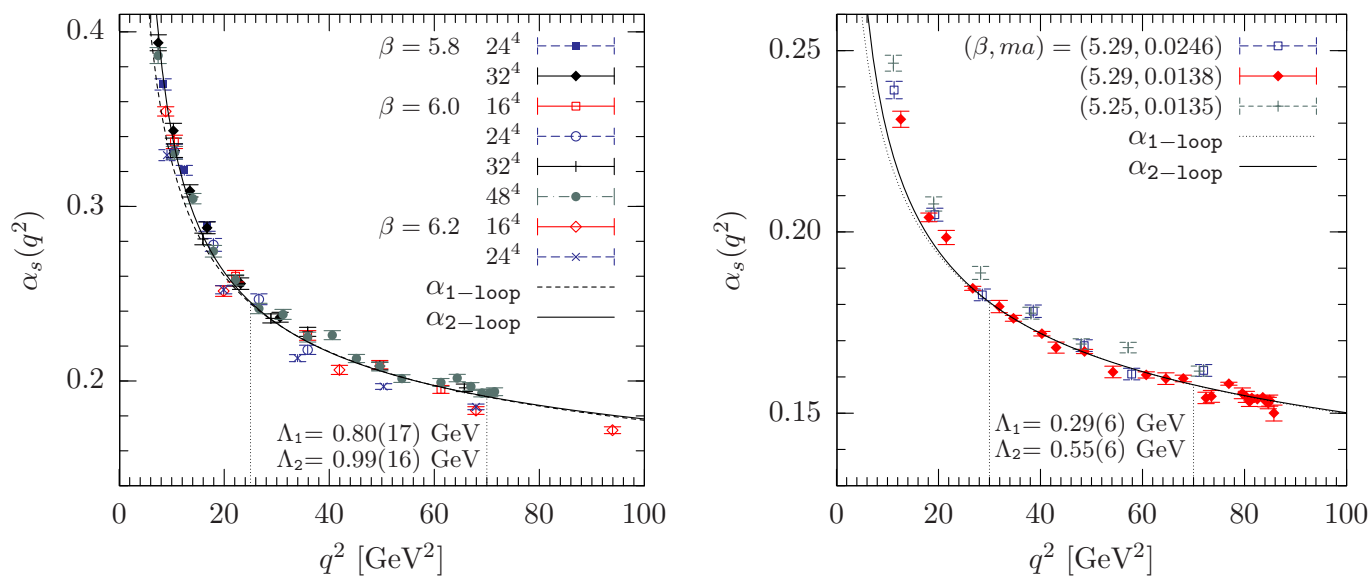

Figure 8: The running coupling $\alpha_{s}$ at large momenta for the quenched (left) and unquenched case (right). Since discretization errors are quite large we used only data from the $48^{4}\left(24^{3} \times 48\right)$ lattice at $\beta=6.0$ ( $\beta=5.29, m a=0.0138$ ) to fit the 1-loop and 2-loop expressions of $\alpha_{s}$ to the data on the left (right) hand side of this figure. 


\section{Conclusions}

We have studied different aspects of lattice Landau gauge theory using MC simulations of quenched and full QCD. Thereby, we have mainly focused on the momentum dependence of the gluon and ghost propagators. We have demonstrated here for the first time that our lattice results for both propagators are consistent with different criteria for confinement, even though we could not confirm the infrared behavior as anticipated from DSE studies. Whether the gluon propagator at zero momentum vanishes in the limit of infinite volume or not has to be clarified in future studies.

We could demonstrate that (dynamical) clover-improved Wilson fermions affect the gluon but only negligibly the ghost propagator. Importantly, the influence of fermions on the infrared behavior of both propagators seems to be small.

On the basis of our present data for $\alpha_{s}$ we cannot confirm it to approach a non-trivial infrared fix-point monotonously from below. In any case, our data at larger momenta agree with the 1-loop and 2-loop expressions of $\alpha_{s}$, even though simulations at larger $\beta$ are worthwhile to perform to suppress discretization effects and to obtain more reliable results for $\Lambda$.

All simulations were performed on the IBM pSeries 690 at HLRN and on the MVS-15000BM at the Joint Supercomputer Center (JSCC) in Moscow. This work was supported by the DFG under the contract FOR 465 (Forschergruppe Lattice Hadron Phenomenology), by the DFG-funded graduate school GK 271 and with joint grants DFG 436 RUS 113/866/0 and RFBR 06-02-04014. We thank the QCDSF collaboration for providing us their unquenched configurations which we could access in the framework of the I3 HadronPhysics initiative (EU contract RII3-CT-2004-506078). We are grateful to Hinnerk Stüben for contributing parts of the program code. A. St. acknowledges discussions with Lorenz von Smekal.

\section{References}

[1] T. Kugo and I. Ojima, Prog. Theor. Phys. Suppl. 66 (1979) 1.

[2] T. Kugo, hep-th/9511033.

[3] L. von Smekal, R. Alkofer and A. Hauck, Phys. Rev. Lett. 79 (1997) 3591-3594, [hep-ph/9705242]; Ann. Phys. 267 (1998) 1, [hep-ph/9707327].

[4] J. M. Pawlowski, D. F. Litim, S. Nedelko and L. von Smekal, Phys. Rev. Lett. 93 (2004) 152002, [hep-th/0312324].

[5] D. Zwanziger, Phys. Rev. D65 (2002) 094039, [hep-th/0109224].

[6] A. Sternbeck, E.-M. Ilgenfritz, M. Müller-Preussker and A. Schiller, Phys. Rev. D72 (2005) 014507, [hep-lat/0506007].

[7] A. Sternbeck, PhD thesis, Humboldt-University Berlin, 2006, hep-lat/0609016.

[8] UKQCD Collaboration, D. B. Leinweber, J. I. Skullerud, A. G. Williams and C. Parrinello, Phys. Rev. D60 (1999) 094507, [hep-lat/9811027].

[9] C. Lerche and L. von Smekal, Phys. Rev. D65 (2002) 125006, [hep-ph/0202194].

[10] P. J. Silva and O. Oliveira, Phys. Rev. D74 (2006) 034513, [hep-lat/0511043]; hep-lat/0609069.

[11] R. Alkofer and L. von Smekal, Phys. Rept. 353 (2001) 281, [hep-ph/0007355].

[12] E.-M. Ilgenfritz, M. Müller-Preussker, A. Sternbeck, A. Schiller and I. L. Bogolubsky, hep-lat/0609043.

[13] A. Cucchieri, T. Mendes and A. Mihara, JHEP 12 (2004) 012, [hep-lat/0408034]; J. C. R. Bloch, A. Cucchieri, K. Langfeld and T. Mendes, Nucl. Phys. B687 (2004) 76-100, [hep-lat/0312036].

[14] W. Schleifenbaum, A. Maas, J. Wambach and R. Alkofer, Phys. Rev. D72 (2005) 014017, [hep-ph/0411052]. 